\title{
Average Structure, Local Structure, Photoluminescence and NLO properties of Scheelite type $\mathrm{NaCe}\left(\mathrm{WO}_{4}\right)_{2}$
}

Archana K. Munirathnappa ${ }^{a, \dagger}$, Joerg C. Neuefeind ${ }^{b}$, Premakumar Yanda ${ }^{c}$, A. Sundaresan ${ }^{c}$, I.V Kityk ${ }^{d}$, K.Ozga ${ }^{d}$, J.Jedrykad, Poornesh Pe, Ashok Rao and Nalini G. Sundaram ${ }^{a}$ *

${ }^{a}$ Materials Science Division, Poornaprajna Institute of Scientific Research, Bidalur, Near Devanahalli562110, Bengaluru, Karnataka, India.

†Manipal Academy of Higher Education, Manipal-576104, Karnataka, India.

${ }^{\mathrm{b}}$ Chemical and Engineering Materials Division, Oak Ridge National Laboratory, Oak Ridge, Tennsssee 37831, United States.

${ }^{\mathrm{c} C h e m i s t r y}$ and Physics of Materials Unit, New Chemistry Unit and School of Advanced Materials, Jawaharlal Nehru Center for Advanced Scientific Research, P.O Bengaluru-560064, Karnataka, India. 'Institute of Optoelectronics and Measuring Systems, Faculty of Electrical Engineering, Czestochowa University of Technology, Armii Krajowej 17, PL-42-201 Czestochowa, Poland.

e Department of Physics, Manipal Institute of Technology, ${ }^{\dagger}$ Manipal Academy of Higher Education, Manipal-576104, Karnataka, India.

*corresponding author: E-mail: nalini@,poornaprajna.org and nalusundaram@gmail.com

\section{Supporting information}

Table S1. Crystallographic data of NaCeW-a obtained by PDF refinements from $r=1.5$ to $50 \AA$ using the structure model obtained from the average structure via Rietveld refinements of RT neutron diffraction data.

\begin{tabular}{l|c}
\hline \multicolumn{1}{c|}{$\begin{array}{c}\text { Crystallographic data } \\
\text { Space group }\end{array}$} & $\begin{array}{c}\mathrm{NaCeW-a} \\
\mathbf{I 4}_{\mathbf{1}} / \mathbf{a}\end{array}$ \\
\hline $\mathbf{a}=\mathbf{b}(\AA)$ & $5.337(2)$ \\
$\mathbf{c}(\AA)$ & $11.628(2)$ \\
Unit cell volume $\left(\AA^{3}\right)$ & $331.35(2)$ \\
$\mathbf{R p}(\%)$ & 9.9 \\
\hline
\end{tabular}

Table S2. Atomic coordinates and thermal parameters of $\mathrm{NaCeW}-\mathrm{a}$ obtained by PDF \begin{tabular}{l|l|l|l}
$\mathrm{NaCeW}-\mathrm{a}$ & Site & Fractional coordinates
\end{tabular}

Occupancy $\quad U_{\text {iso }}\left(\AA^{2}\right)$

refinements in $\mathrm{r}$ range from 1.5 to $50 \mathrm{~A}$ using structure model obtained from the average structure via Rietveld refinements using RT neutron diffraction data. 


\begin{tabular}{|c|c|c|c|c|c|c|}
\hline $\begin{array}{c}\text { Atom site } \\
\text { label }\end{array}$ & & $x$ & $y$ & $z$ & & \\
\hline Ce1 & $2 b$ & 0.000 & 0.500 & 0.750 & $0.5(1)$ & $0.011(1)$ \\
\hline $\mathrm{Ce} 2$ & $2 b$ & 0.500 & 0.000 & 0.250 & $0.5(1)$ & $0.030(1)$ \\
\hline Na1 & $2 b$ & 0.000 & 0.500 & 0.750 & $0.5(1)$ & $0.030(1)$ \\
\hline $\mathrm{Na} 2$ & $2 b$ & 0.500 & 0.000 & 0.250 & $0.5(1)$ & $0.011(1)$ \\
\hline $\mathrm{Ce} 3$ & $2 d$ & 0.500 & 0.500 & 0.000 & $0.5(1)$ & $0.030(1)$ \\
\hline Ce4 & $2 d$ & 0.000 & 0.000 & 0.500 & $0.5(1)$ & $0.011(1)$ \\
\hline $\mathrm{Na3}$ & $2 d$ & 0.500 & 0.500 & 0.000 & $0.5(1)$ & $0.011(1)$ \\
\hline $\mathrm{Na} 4$ & $2 d$ & 0.000 & 0.000 & 0.500 & $0.5(1)$ & $0.030(1)$ \\
\hline W1 & $2 \mathrm{a}$ & 0.000 & 0.000 & 0.000 & $1(1)$ & 0.009 (1) \\
\hline W2 & $2 a$ & 0.500 & 0.500 & 0.500 & $1(1)$ & $0.011(1)$ \\
\hline W3 & $2 c$ & 0.500 & 0.000 & 0.750 & $1(1)$ & $0.011(1)$ \\
\hline W4 & $2 c$ & 0.000 & 0.500 & 0.250 & $1(1)$ & 0.009 (1) \\
\hline 01 & $8 \mathrm{~g}$ & $0.7444(1)$ & $0.8964(1)$ & $0.5146(1)$ & $1(1)$ & $0.024(1)$ \\
\hline O2 & $8 \mathrm{~g}$ & $0.2551(1)$ & 0.6035 (1) & $0.5416(1)$ & $1(1)$ & $0.025(1)$ \\
\hline $\mathbf{O 3}$ & $8 \mathrm{~g}$ & $0.1475(1)$ & $0.5163(1)$ & $0.7901(1)$ & $1(1)$ & 0.019 (1) \\
\hline 04 & $8 \mathrm{~g}$ & 0.8524 (1) & $0.9836(1)$ & 0.7901 (1) & $1(1)$ & 0.019 (1) \\
\hline 05 & $8 \mathrm{~g}$ & $0.2661(1)$ & $0.1024(1)$ & 0.4595 (1) & $1(1)$ & $0.016(1)$ \\
\hline 06 & $8 \mathrm{~g}$ & $0.7338(1)$ & $0.3975(1)$ & $0.4595(1)$ & $1(1)$ & $0.016(1)$ \\
\hline 07 & $8 \mathrm{~g}$ & $0.8535(1)$ & $0.4939(1)$ & $0.2082(1)$ & $1(1)$ & $0.014(1)$ \\
\hline 08 & $8 g$ & 0.1464 (1) & $0.0060(1)$ & $0.2082(1)$ & $1(1)$ & 0.014 (1) \\
\hline O9 & $8 \mathrm{~g}$ & $0.2440(1)$ & $0.3964(1)$ & $0.0140(1)$ & $1(1)$ & $0.024(1)$ \\
\hline 010 & $8 \mathrm{~g}$ & $0.7555(1)$ & 0.1035 & $0.0140(1)$ & $1(1)$ & 0.025 (1) \\
\hline 011 & $8 \mathrm{~g}$ & $0.6475(1)$ & $0.0163(1)$ & 0.2901 (1) & $1(1)$ & 0.019 (1) \\
\hline 012 & $8 \mathrm{~g}$ & $0.3524(1)$ & $0.4836(1)$ & $0.2901(1)$ & $1(1)$ & 0.019 (1) \\
\hline 013 & $8 \mathrm{~g}$ & $0.7661(1)$ & $0.6024(1)$ & 0.9595 (1) & $1(1)$ & $0.016(1)$ \\
\hline 014 & $8 \mathrm{~g}$ & $0.2338(1)$ & $0.8975(1)$ & $0.9596(1)$ & $1(1)$ & $0.016(1)$ \\
\hline 015 & $8 \mathrm{~g}$ & $0.3535(1)$ & $0.9939(1)$ & $0.7082(1)$ & $1(1)$ & $0.014(1)$ \\
\hline 016 & $8 \mathrm{~g}$ & 0.6464 (1) & $0.5060(1)$ & $0.7082(1)$ & $1(1)$ & 0.014 (1) \\
\hline
\end{tabular}


Table S3. Crystallographic data of NaCeW-a obtained from the PDF refinements using $I \overline{4}$ model and $I 4_{1} /$ a model from $\mathrm{r}=1.5$ to $10 \AA$.

\begin{tabular}{l|c|c}
\hline \multirow{1}{*}{$\begin{array}{c}\text { Crystallographic data } \\
\text { Space group }\end{array}$} & \multicolumn{2}{c}{ NaCeW-a } \\
\cline { 2 - 3 } & $\boldsymbol{I} \overline{\mathbf{4}}$ & $\boldsymbol{I 4}_{\boldsymbol{I}} / \mathbf{a}$ \\
\hline $\mathbf{a}=\mathbf{b}(\AA)$ & $5.326(2)$ & $5.345(3)$ \\
$\mathbf{c}(\boldsymbol{\AA})$ & $11.431(2)$ & $11.651(2)$ \\
Unit cell volume $\left(\AA^{3}\right)$ & $324.25(2)$ & $332.82(1)$ \\
$\mathbf{R}_{\mathbf{W}}(\%)$ & 13.4 & 20.9 \\
\hline
\end{tabular}

Table S4. Crystallographic data of NaCeW-b obtained from the PDF refinements using $I \overline{4}$ model and $I 4_{1} /$ a model from $\mathrm{r}=1.5$ to $3.5 \AA$.

\begin{tabular}{l|c|c}
\hline \multirow{2}{*}{$\begin{array}{c}\text { Crystallographic data } \\
\text { Space group }\end{array}$} & \multicolumn{2}{c}{ NaCeW-b } \\
\cline { 2 - 3 } & $\boldsymbol{I} \overline{\mathbf{4}}$ & $\boldsymbol{I 4}_{\boldsymbol{I}} / \mathbf{a}$ \\
\hline $\mathbf{a}=\mathbf{b}(\AA)$ & $5.329(1)$ & $5.448(1)$ \\
$\mathbf{c}(\AA)$ & $11.481(1)$ & $11.405(1)$ \\
Unit cell volume $\left(\AA^{3}\right)$ & $325.86(1)$ & $338.5(1)$ \\
$\mathbf{R}_{\mathbf{W}}(\%)$ & 3.9 & 10.7 \\
\hline
\end{tabular}


Table S5a. Atomic coordinates and thermal parameters obtained from the PDF refinements of $\mathrm{NaCeW}$-a using the $I \overline{4}$ model from $\mathrm{r}=1.5$ to $3.5 \AA$.

\begin{tabular}{|c|c|c|c|c|c|c|}
\hline \multirow{2}{*}{$\begin{array}{c}\text { Atom site } \\
\text { label }\end{array}$} & \multirow[t]{2}{*}{ Site } & \multicolumn{3}{|c|}{ Fractional coordinates } & \multirow[t]{2}{*}{ Occupancy } & \multirow[t]{2}{*}{$U_{\text {iso }}\left(\AA^{2}\right)$} \\
\hline & & $x$ & $y$ & $z$ & & \\
\hline Ce1 & $2 b$ & 0.000 & 0.500 & 0.750 & $0.51(2)$ & $0.007(1)$ \\
\hline $\mathrm{Ce} 2$ & $2 b$ & 0.500 & 0.000 & 0.250 & $0.51(2)$ & $0.007(1)$ \\
\hline Na1 & $2 b$ & 0.000 & 0.500 & 0.750 & $0.48(2)$ & $0.007(1)$ \\
\hline $\mathrm{Na} 2$ & $2 b$ & 0.500 & 0.000 & 0.250 & $0.48(2)$ & $0.007(1)$ \\
\hline Ce3 & $2 d$ & 0.500 & 0.500 & 0.000 & $0.51(2)$ & $0.001(1)$ \\
\hline Ce4 & $2 d$ & 0.000 & 0.000 & 0.500 & $0.51(2)$ & $0.001(1)$ \\
\hline $\mathrm{Na3}$ & $2 \mathrm{~d}$ & 0.500 & 0.500 & 0.000 & $0.48(2)$ & $0.001(1)$ \\
\hline $\mathrm{Na} 4$ & $2 \mathrm{~d}$ & 0.000 & 0.000 & 0.500 & $0.48(2)$ & $0.001(1)$ \\
\hline W1 & $2 \mathrm{a}$ & 0.000 & 0.000 & 0.000 & $1(1)$ & $0.002(1)$ \\
\hline W2 & $2 a$ & 0.500 & 0.500 & 0.500 & $1(1)$ & $0.002(1)$ \\
\hline W3 & $2 \mathrm{c}$ & 0.500 & 0.000 & 0.750 & $1(1)$ & $0.002(1)$ \\
\hline W4 & $2 \mathrm{c}$ & 0.000 & 0.500 & 0.250 & $1(1)$ & $0.002(1)$ \\
\hline 01 & $8 \mathrm{~g}$ & 0.1598 (1) & $0.2387(1)$ & 0.9199 (1) & $1(1)$ & $0.009(1)$ \\
\hline 02 & $8 \mathrm{~g}$ & 0.8401 (1) & 0.7614 (1) & 0.9199 (1) & $1(1)$ & $0.009(1)$ \\
\hline $\mathbf{0 3}$ & $8 g$ & $0.7612(1)$ & $0.1598(1)$ & $0.0800(1)$ & $1(1)$ & $0.019(1)$ \\
\hline O4 & $8 \mathrm{~g}$ & $0.2397(1)$ & $0.8401(1)$ & $0.0800(1)$ & $1(1)$ & $0.019(1)$ \\
\hline 05 & $8 \mathrm{~g}$ & 0.6598 (1) & 0.7387 (1) & 0.4199 (1) & $1(1)$ & $0.009(1)$ \\
\hline 06 & $8 \mathrm{~g}$ & $0.3401(1)$ & $0.2612(1)$ & 0.4199 (1) & $1(1)$ & $0.009(1)$ \\
\hline 07 & $8 g$ & $0.2612(1)$ & 0.6598 (1) & $0.5800(1)$ & $1(1)$ & $0.019(1)$ \\
\hline 08 & $8 \mathrm{~g}$ & $0.7387(1)$ & $0.3401(1)$ & $0.5800(1)$ & $1(1)$ & $0.019(1)$ \\
\hline O9 & $8 \mathrm{~g}$ & $0.6583(1)$ & $0.2521(1)$ & $0.8251(1)$ & $1(1)$ & $0.014(1)$ \\
\hline 010 & $8 \mathrm{~g}$ & 0.3417 (1) & 0.7578 (1) & 0.8251 (1) & $1(1)$ & $0.014(1)$ \\
\hline 011 & $8 \mathrm{~g}$ & $0.7529(1)$ & $0.6583(1)$ & $0.1748(1)$ & $1(1)$ & $0.040(1)$ \\
\hline 012 & $8 \mathrm{~g}$ & $0.2529(1)$ & $0.3417(1)$ & $0.1748(1)$ & $1(1)$ & $0.040(1)$ \\
\hline 013 & $8 \mathrm{~g}$ & $0.1581(1)$ & $0.7521(1)$ & $0.3251(1)$ & $1(1)$ & $0.014(1)$ \\
\hline 014 & $8 \mathrm{~g}$ & 0.8418 (1) & $0.2478(1)$ & $0.3251(1)$ & $1(1)$ & $0.014(1)$ \\
\hline 015 & $8 g$ & 0.2478 (1) & 0.1581 (1) & 0.6748 & $1(1)$ & $0.040(1)$ \\
\hline
\end{tabular}




\begin{tabular}{l|l|lll|l|l}
\hline $\mathbf{0 1 6}$ & $8 \mathrm{~g}$ & $0.7421(1)$ & $0.8418(1)$ & $0.6748(1)$ & $1(1)$ & $0.040(1)$ \\
\hline
\end{tabular}




\begin{tabular}{|c|c|c|c|c|c|c|}
\hline \multirow[t]{2}{*}{ Atom label } & \multirow[t]{2}{*}{ Site } & \multicolumn{3}{|c|}{ Fractional coordinates } & \multirow[t]{2}{*}{ Occupancy } & \multirow[t]{2}{*}{$U_{i s o}\left(\AA^{2}\right)$} \\
\hline & & $\mathbf{x}$ & $\mathbf{y}$ & $\mathbf{z}$ & & \\
\hline Ce1 & $2 b$ & 0.000 & 0.500 & 0.750 & $0.51(2)$ & $0.006(1)$ \\
\hline $\mathrm{Ce} 2$ & $2 b$ & 0.500 & 0.000 & 0.250 & $0.51(2)$ & $0.006(1)$ \\
\hline Na1 & $2 b$ & 0.000 & 0.500 & 0.750 & $0.48(2)$ & $0.006(1)$ \\
\hline $\mathrm{Na} 2$ & $2 b$ & 0.500 & 0.000 & 0.250 & $0.48(2)$ & $0.006(1)$ \\
\hline Ce3 & $2 \mathrm{~d}$ & 0.500 & 0.500 & 0.000 & $0.51(2)$ & $0.001(1)$ \\
\hline Ce4 & $2 \mathrm{~d}$ & 0.000 & 0.000 & 0.500 & $0.51(2)$ & $0.001(1)$ \\
\hline $\mathrm{Na3}$ & $2 \mathrm{~d}$ & 0.500 & 0.500 & 0.000 & $0.48(2)$ & $0.001(1)$ \\
\hline $\mathrm{Na} 4$ & $2 \mathrm{~d}$ & 0.000 & 0.000 & 0.500 & $0.48(2)$ & $0.001(1)$ \\
\hline W1 & $2 \mathrm{a}$ & 0.000 & 0.000 & 0.000 & $1(1)$ & $0.007(1)$ \\
\hline W2 & $2 \mathrm{a}$ & 0.500 & 0.500 & 0.500 & $1(1)$ & $0.007(1)$ \\
\hline W3 & $2 \mathrm{c}$ & 0.500 & 0.000 & 0.750 & $1(1)$ & $0.012(1)$ \\
\hline W4 & $2 \mathrm{c}$ & 0.000 & 0.500 & 0.250 & $1(1)$ & $0.012(1)$ \\
\hline 01 & $8 \mathrm{~g}$ & $0.1600(1)$ & $0.2461(1)$ & $0.9209(1)$ & $1(1)$ & $0.010(1)$ \\
\hline $\mathrm{O} 2$ & $8 \mathrm{~g}$ & $0.8396(1)$ & 0.7538 & $0.9209(1)$ & $1(1)$ & $0.010(1)$ \\
\hline $\mathbf{O 3}$ & $8 \mathrm{~g}$ & 0.7538 & 0.1603 & 0.0793 & $1(1)$ & $0.023(1)$ \\
\hline O4 & $8 g$ & $0.2461(1)$ & $0.8396(1)$ & 0.0793 (1) & $1(1)$ & $0.023(1)$ \\
\hline 05 & $8 \mathrm{~g}$ & 0.6603 & $0.7461(1)$ & 0.4269 (1) & $1(1)$ & $0.010(1)$ \\
\hline 06 & $8 \mathrm{~g}$ & $0.3396(1)$ & 0.2538 & 0.4269 (1) & $1(1)$ & $0.010(1)$ \\
\hline O7 & $8 g$ & 0.2538 & 0.6603 & $0.5793(1)$ & $1(1)$ & $0.023(1)$ \\
\hline 08 & $8 \mathrm{~g}$ & $0.7461(1)$ & $0.3396(1)$ & $0.5793(1)$ & $1(1)$ & $0.023(1)$ \\
\hline O9 & $8 \mathrm{~g}$ & $0.6583(1)$ & $0.2477(1)$ & $0.8258(1)$ & $1(1)$ & $0.011(1)$ \\
\hline 010 & $8 g$ & $0.3417(1)$ & 0.7522 & $0.8258(1)$ & $1(1)$ & $0.011(1)$ \\
\hline 011 & $8 g$ & 0.7522 & 0.6582 & $0.1741(1)$ & $1(1)$ & $0.031(1)$ \\
\hline 012 & $8 \mathrm{~g}$ & $0.2457(1)$ & $0.3417(1)$ & $0.1741(1)$ & $1(1)$ & $0.031(1)$ \\
\hline 013 & $8 \mathrm{~g}$ & $0.1582(1)$ & $0.7477(1)$ & $0.3258(1)$ & $1(1)$ & $0.011(1)$ \\
\hline 014 & $8 \mathrm{~g}$ & $0.8417(1)$ & $0.2522(1)$ & $0.3258(1)$ & $1(1)$ & $0.011(1)$ \\
\hline 015 & $8 \mathrm{~g}$ & $0.2522(1)$ & 0.1582 & 0.6741 (1) & $1(1)$ & $0.031(1)$ \\
\hline 016 & $8 \mathrm{~g}$ & 0.7477 & $0.8417(1)$ & $0.6741(1)$ & $1(1)$ & $0.031(1)$ \\
\hline
\end{tabular}


Table S6. Selected bond distances in $\mathrm{NaCeW}-\mathrm{a}$ and $\mathrm{NaCeW}-\mathrm{b}$ via PDF refinements from $\mathrm{r}=1.5$ to 3.5 Å using $I \overline{4}$ model.

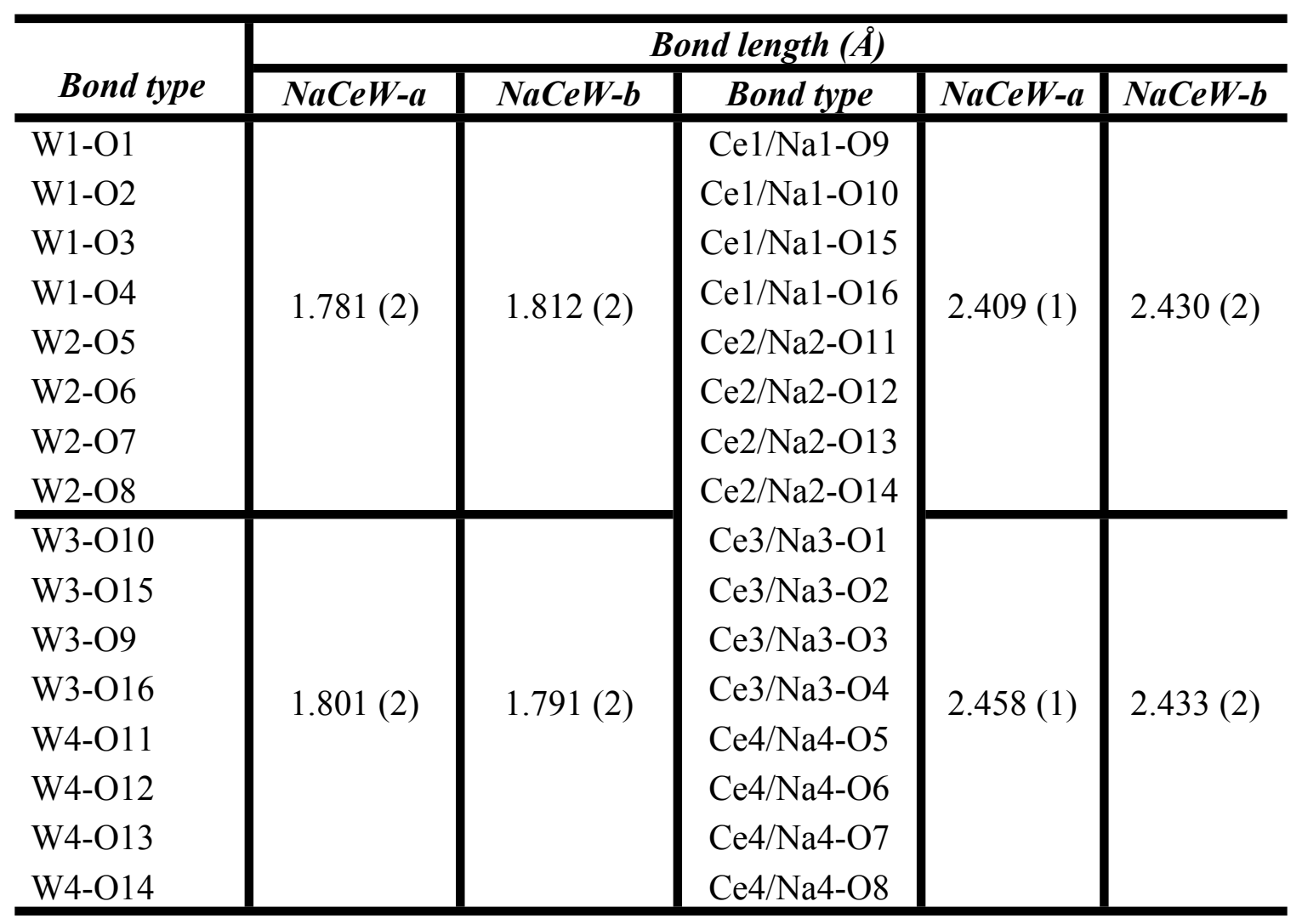


Table S7. Selected bond angle in $\mathrm{NaCeW}-\mathrm{a}$ and $\mathrm{NaCeW}-\mathrm{b}$ via PDF refinements from $\mathrm{r}=1.5$ to $3.5 \AA$ using $I \overline{4}$ model.

\begin{tabular}{l|l|l}
\hline \multirow{2}{*}{ Bond angle type } & \multicolumn{2}{|c}{ Bond angle () } \\
\hline & NaCeW-a & NaCeW-b \\
\hline O1-W1-Ce3/Na3 & \\
O2-W1-Ce3/Na3 & \\
O3-W1-Ce3/Na3 & \\
O4-W1-Ce3/Na3 & $124.27(2)$ & \\
\hline O5-W2-Ce4/Na4 & \\
O6-W2-Ce4/Na4 & \\
O7-W2-Ce4/Na4 & \\
O8-W2-Ce4/Na4 & \\
\hline O9-W3-Ce1/Na1 & \\
O10-W3-Ce1/Na1 & \\
O11-W3-Ce1/Na1 & \\
O12-W3-Ce1/Na1 & \\
\hline O13-W4-Ce2/Na2 & \\
O14-W4-Ce2/Na2 & \\
O15-W4-Ce2/Na2 & \\
O16-W4-Ce2/Na2 & \\
\hline
\end{tabular}

Table S8. Measured polyhedral volume, distortion indexes and Bond valence sum of NaCeW-a and $\mathrm{NaCeW}-\mathrm{b}$ from the Rietveld refinement method $\left(I 4_{1} / \mathrm{a}\right)$.

\begin{tabular}{|c|c|c|c|c|c|c|c|}
\hline \multirow{2}{*}{$\begin{array}{l}\text { SL. } \\
\text { No. }\end{array}$} & \multirow{2}{*}{$\begin{array}{c}\text { Type of tetrahedra } \\
\text { /polyhedra }\end{array}$} & \multicolumn{2}{|c|}{ Polyhedral volume $\left(\AA^{3}\right)$} & \multicolumn{2}{|c|}{ Distortion Indexes } & \multicolumn{2}{|c|}{ BVS } \\
\hline & & NaCeW-a & NaCeW-b & NaCeW-a & NaCeW-b & NaCeW-a & $\mathbf{N a C e W}-\mathbf{b}$ \\
\hline $\begin{array}{l}1 . \\
2 .\end{array}$ & $\begin{array}{l}\mathrm{WO}_{4} \\
\mathrm{Na} / \mathrm{CeO}_{8}\end{array}$ & $\begin{array}{l}2.667 \\
29.32\end{array}$ & $\begin{array}{l}2.915 \\
28.66\end{array}$ & $\begin{array}{l}0.000 \\
0.009\end{array}$ & $\begin{array}{l}0.000 \\
0.003\end{array}$ & $\begin{array}{l}6.15 \\
2.41\end{array}$ & $\begin{array}{l}5.42 \\
2.52\end{array}$ \\
\hline
\end{tabular}


Table S9. Measured polyhedral volume, distortion indexes and bond valence sum of $\mathrm{NaCeW}-\mathrm{a}$ and $\mathrm{NaCeW}-\mathrm{b}$ from the PDF analysis in the range $\mathrm{r}=1.5$ to $3.5 \AA$.

\begin{tabular}{|c|c|c|c|c|c|c|c|}
\hline \multirow{2}{*}{$\begin{array}{l}\text { SL. } \\
\text { No. }\end{array}$} & \multirow{2}{*}{$\begin{array}{c}\text { Type of } \\
\text { tetrahedra/polyhedra }\end{array}$} & \multicolumn{2}{|c|}{ Polyhedral volume $\left(\AA^{3}\right)$} & \multicolumn{2}{|c|}{ Distortion Indexes } & \multicolumn{2}{|c|}{ BVS } \\
\hline & & $\mathrm{NaCeW}-\mathrm{a}$ & $\mathrm{NaCeW}-\mathrm{b}$ & NaCeW-a & NaCeW-b & NaCeW-a & NaCeW-b \\
\hline $\begin{array}{l}1 . \\
2 . \\
3 . \\
4 .\end{array}$ & $\begin{array}{l}{\mathrm{W} 1 O_{4}}{\mathrm{~W} 2 O_{4}} \\
\mathrm{~W} \mathrm{O}_{4} \\
\mathrm{~W}_{4} \mathrm{O}_{4}\end{array}$ & $\begin{array}{l}2.85 \\
2.85 \\
2.87 \\
2.87\end{array}$ & $\begin{array}{l}2.97 \\
2.97 \\
2.85 \\
2.85\end{array}$ & $\begin{array}{l}0.000 \\
0.000 \\
0.000 \\
0.000\end{array}$ & $\begin{array}{l}0.000 \\
0.000 \\
0.000 \\
0.000\end{array}$ & $\begin{array}{l}5.50 \\
5.50 \\
5.21 \\
5.21\end{array}$ & $\begin{array}{l}5.07 \\
5.07 \\
5.35 \\
5.35\end{array}$ \\
\hline $\begin{array}{l}5 . \\
6 . \\
7 . \\
8 .\end{array}$ & $\begin{array}{l}\mathrm{Na} 1 / \mathrm{Ce} 1 \mathrm{O}_{8} \\
\mathrm{Na} 2 / \mathrm{Ce} 2 \mathrm{O}_{8} \\
\mathrm{Na} 3 / \mathrm{Ce} 3 \mathrm{O}_{8} \\
\mathrm{Na} 4 / \mathrm{Ce} 4 \mathrm{O}_{8}\end{array}$ & $\begin{array}{l}26.75 \\
26.75 \\
27.26 \\
27.62\end{array}$ & $\begin{array}{l}27.08 \\
27.08 \\
27.47 \\
27.47\end{array}$ & $\begin{array}{l}0.025 \\
0.025 \\
0.014 \\
0.014\end{array}$ & $\begin{array}{l}0.020 \\
0.020 \\
0.022 \\
0.022\end{array}$ & $\begin{array}{l}2.91 \\
2.97 \\
2.75 \\
2.75\end{array}$ & $\begin{array}{l}2.89 \\
2.89 \\
2.78 \\
2.78\end{array}$ \\
\hline
\end{tabular}

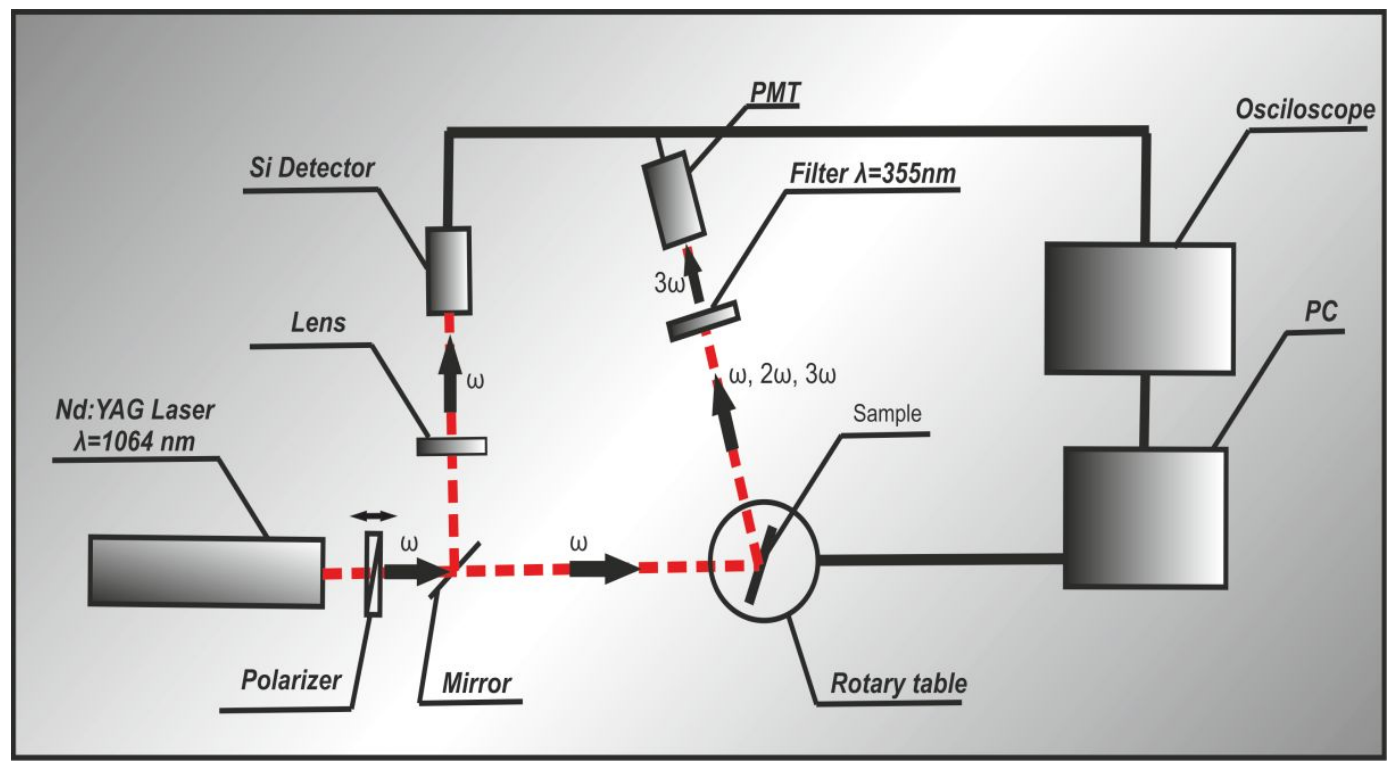

Figure S1. Principal set-up for the SHG measurements in the photo induced regime. 

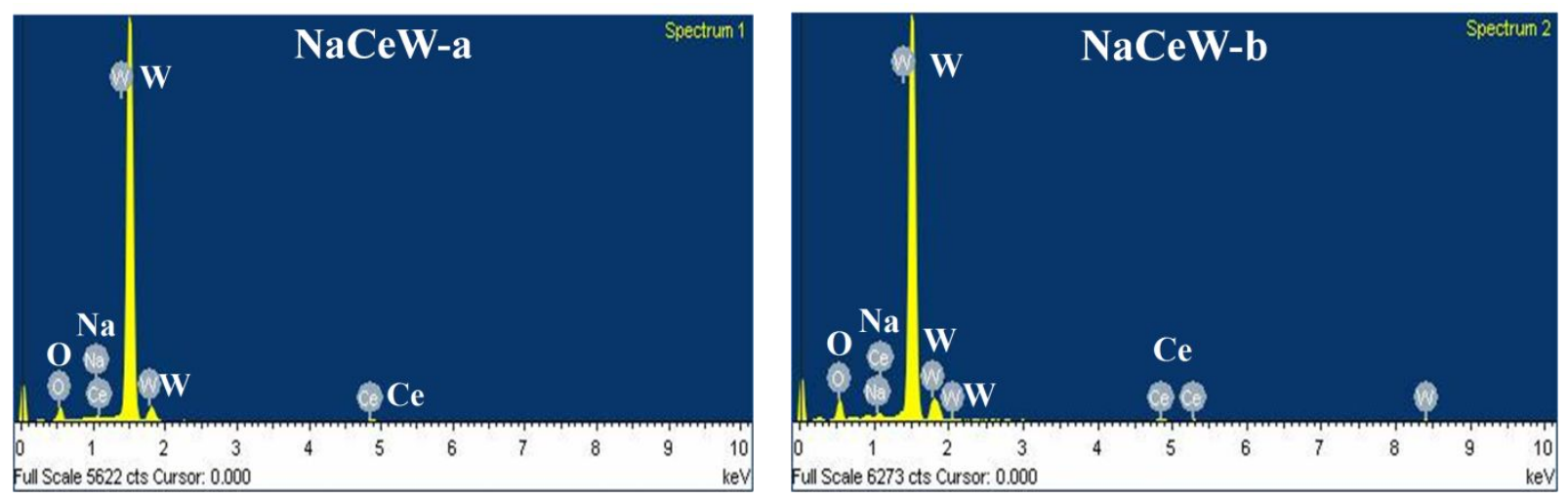

Figure S2. EDS spectra representing characteristic elemental compositions of $\mathrm{NaCe}\left(\mathrm{WO}_{4}\right)_{2}$ determined by FESEM measurements for both $\mathrm{NaCeW}$-a and $\mathrm{NaCeW}$-b.

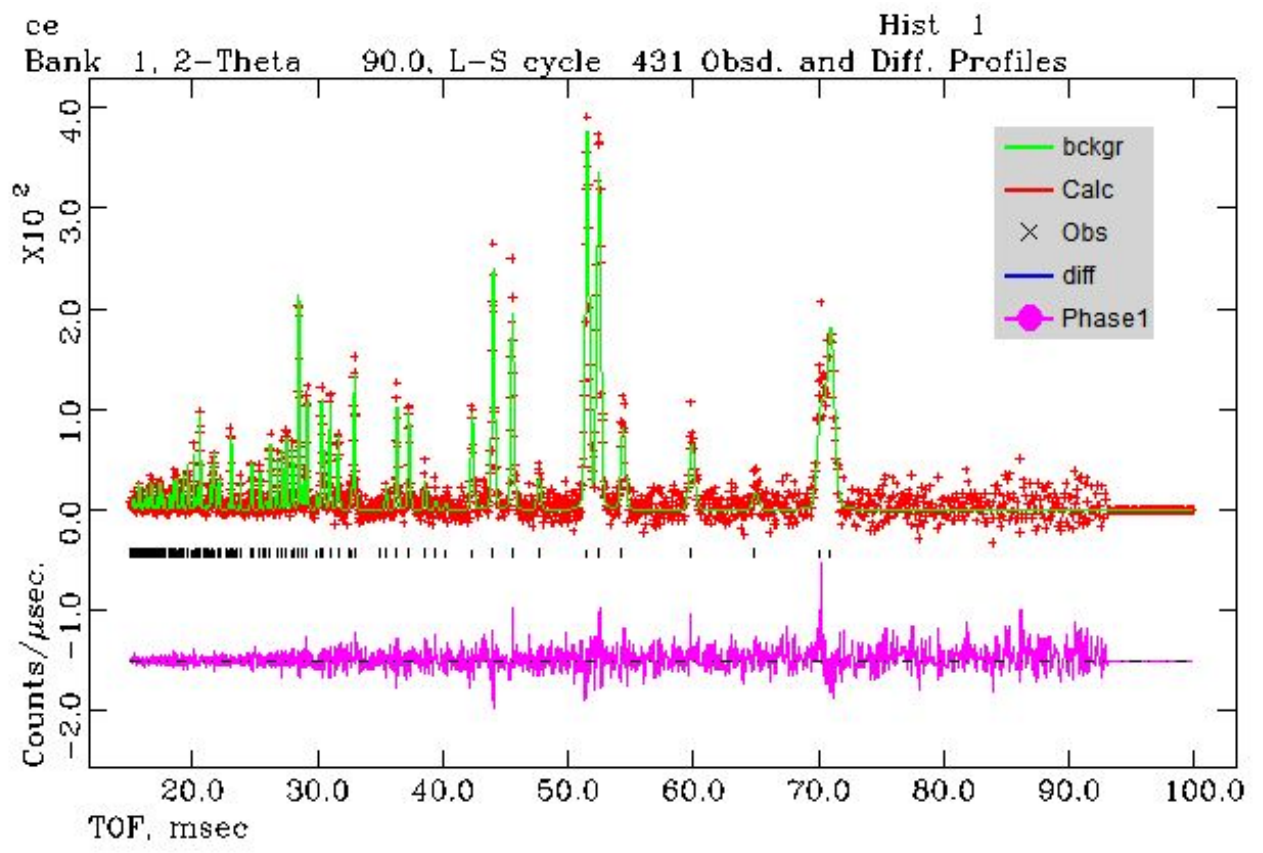

Figure S3. Observed, calculated and difference plot obtained from the Rietveld refinements using RT neutron diffraction data of $\mathrm{NaCeW}$-a collected on POWGEN instrument. 

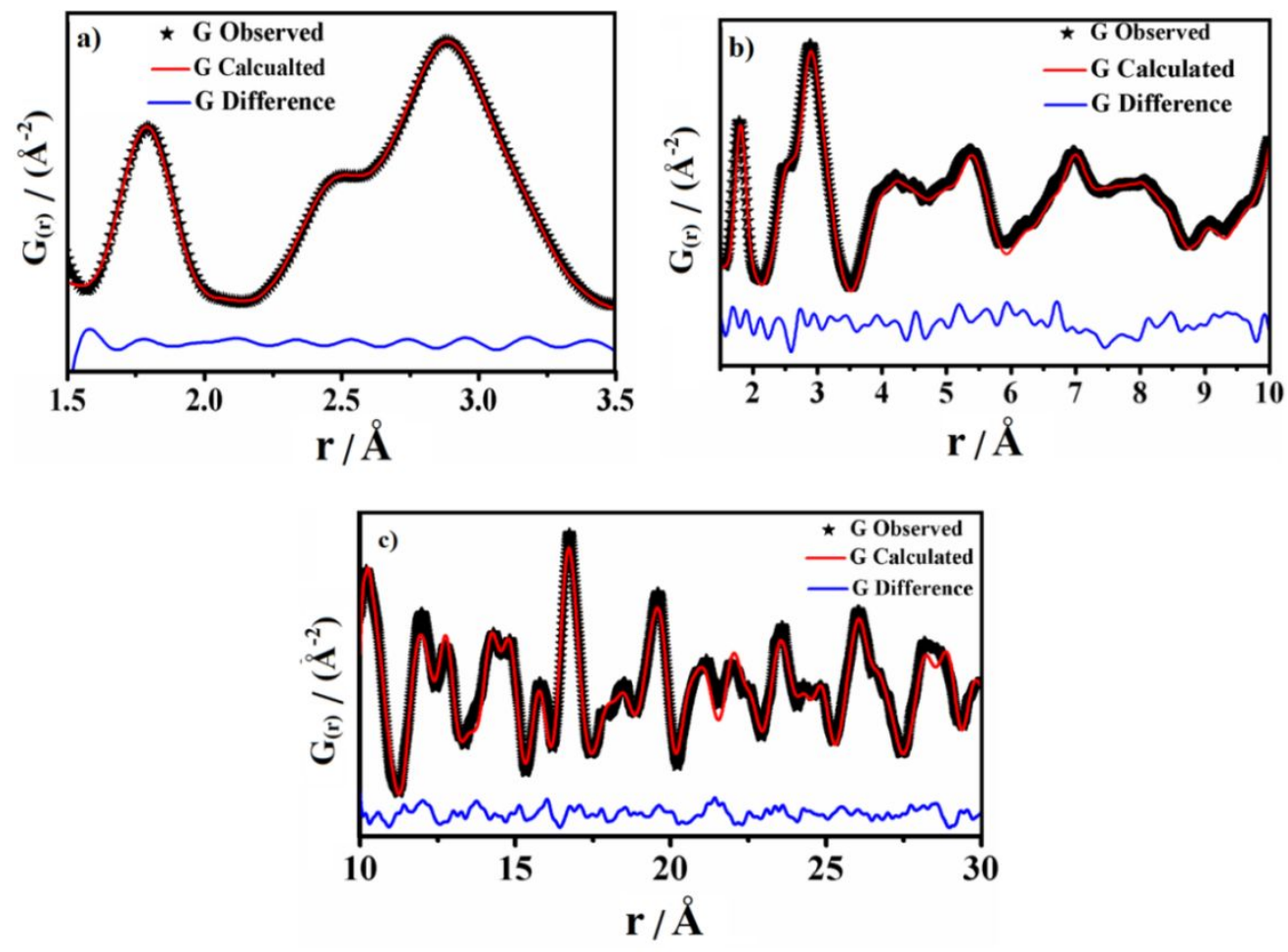

Figure S4. Observed (black stars), calculated (red), and difference (blue) PDF plot of NaCeW-b in various r-ranges using $I \overline{4}$ space group model a) $1.5-3.5 \AA$ b) $1.5-10 \AA$ and c) $10-30 \AA$ using $I 4_{1} / \mathrm{a}$ space group model, suggesting that the material adopts $I \overline{4}$ locally below $10 \AA$ however it adopts $I 4_{1} /$ a model in the long range order.

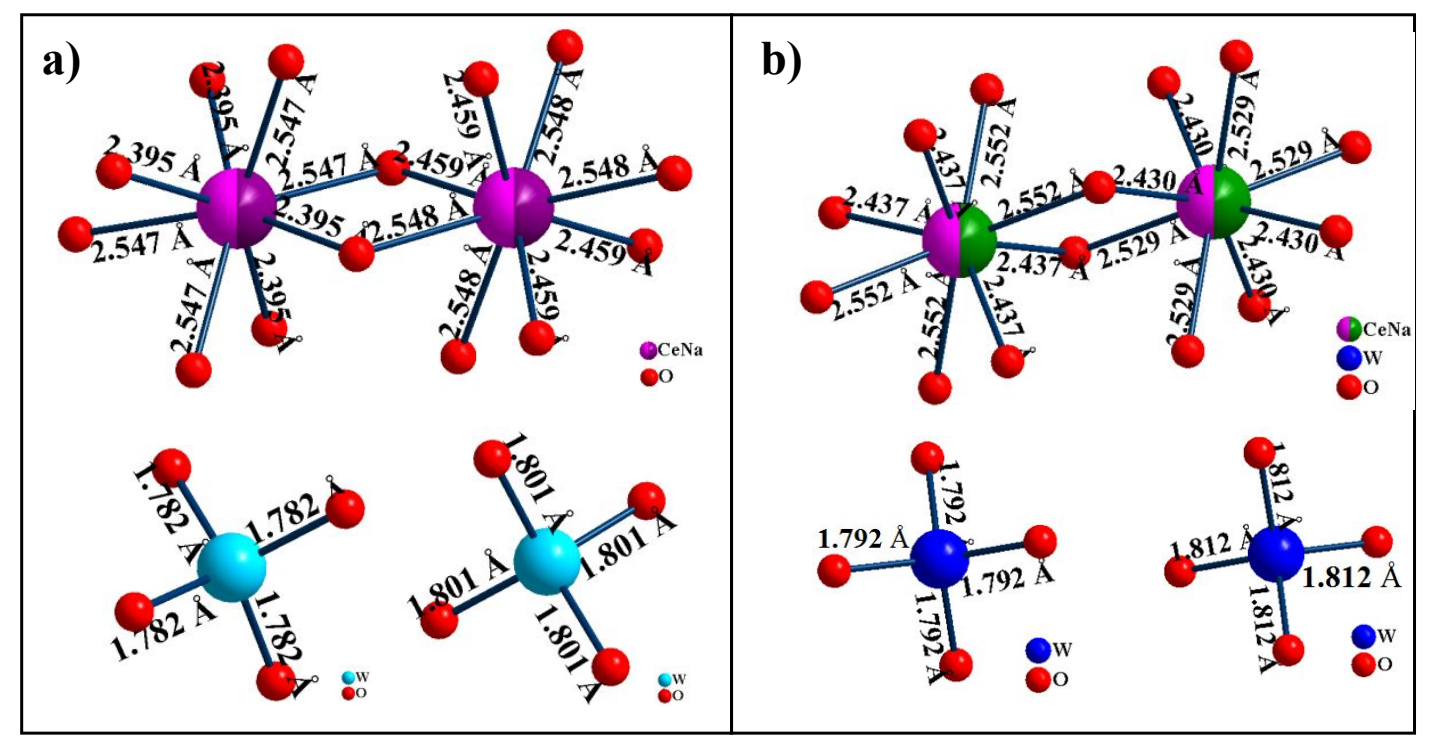

Figure S5. Schematic representation of two different kinds of $\mathrm{Na} / \mathrm{Ce}-\mathrm{O}_{8}$ and $\mathrm{WO}_{4}$ in $\mathrm{NaCeW}-\mathrm{a}$ and NaCeW-b with two types of bond lengths derived from the PDF analysis using $I \overline{4}$ space group. 


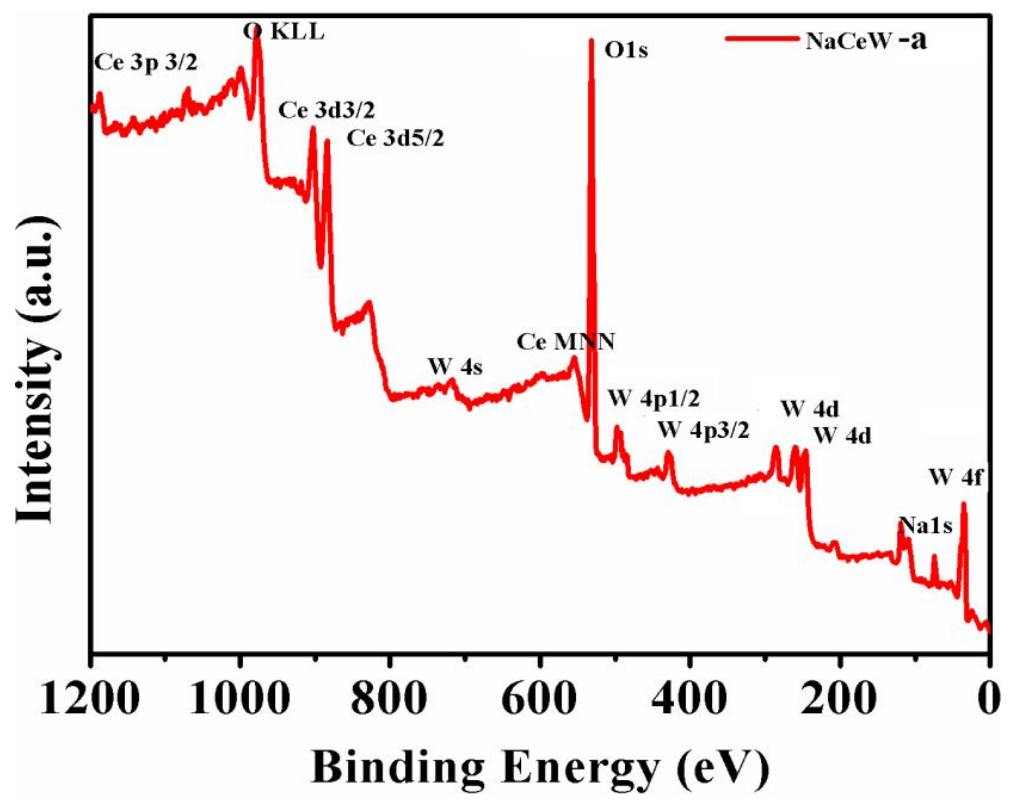

Figure S6. XPS wide spectrum of Ce-3d, W-4f, O $1 s$ and $\mathrm{Na} 1 s$ of as prepared NaCeW-a synthesized under hydrothermal condition at $200{ }^{\circ} \mathrm{C}$.
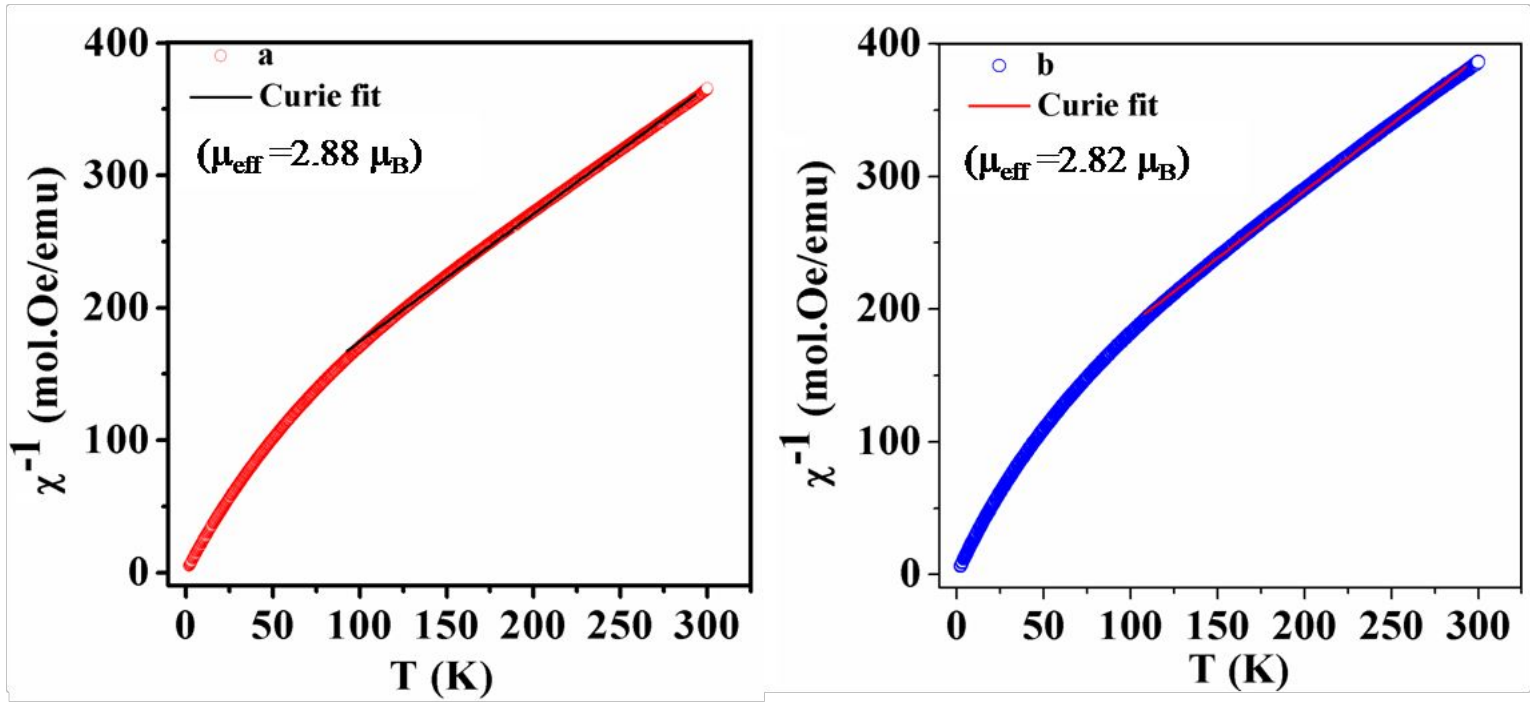

Figure S7. Temperature dependent inverse magnetic susceptibility against temperature of a) $\mathrm{NaCeW}-\mathrm{a}$ and b) $\mathrm{NaCeW}-\mathrm{b}$ profile fit using Curie-Weiss law. 


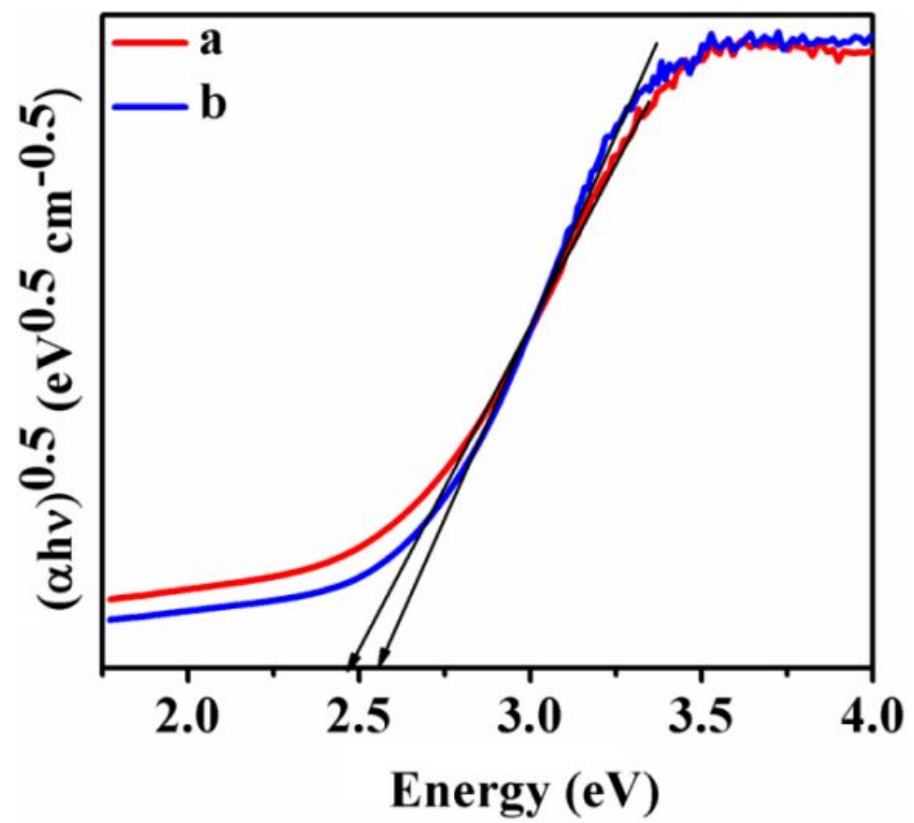

Figure S8. Tauc plot of a) NaCeW-a and b) NaCeW-b obtained using diffuse reflectance studies via Kubelka-Munk function. 